\title{
TELERADIOLOGY- EASY ACCESSIBILITY OF RADIOLOGICAL SERVICES FOR DIAGNOSIS- OUR EXPERIENCE
}

\author{
Murali Belman', Mandapal Toka², Nagendra Thanugondra Raghupathi', Prasad S. G. ${ }^{4}$, Arun Kumar A5, Venkatakrishna Satram ${ }^{6}$, \\ Bhavan Kumar $M^{7}$, Sandilya Biruduraju ${ }^{8}$
}

\author{
${ }_{1}^{1}$ Associate Professor, Department of Radiology and Imaging, Bhaskar Medical College, Hyderabad, Telangana, India. \\ 2Professor, Department of Radiology and Imaging, Bhaskar Medical College, Hyderabad, Telangana, India. \\ ${ }^{3} \mathrm{HOD}$, Department of Radiology, Care Foundation, Hyderabad, Telangana, India. \\ ${ }^{4}$ Chief of Telemedicine, Care Foundation, Hyderabad, Telangana, India. \\ ${ }_{5}^{5}$ Project Manager, Care Foundation, Hyderabad, Telangana, India. \\ ${ }^{6}$ Network Manager, Care Foundation, Hyderabad, Telangana, India. \\ 7 Project Engineer, Care Foundation, Hyderabad, Telangana, India. \\ ${ }^{8}$ Admin-Tele Imaging, Care Foundation, Hyderabad, Telangana, India.
}

\section{BACKGROUND}

\section{ABSTRACT}

Diagnostic imaging is one of the most revolutionary innovations in the medical world. It has revolutionized how physicians and patients view health and diseases. However, the dearth of radiologists to read these images has been an impending problem worldwide. Digitization of medical images in standard Digital Imaging and Communications in Medicine (DICOM) format and the seamless integration of imaging modalities through the Picture Archiving and Communication System (PACS) has enabled easy acquisition and transfer of images across networks for viewing, enabling the teleradiology platform for reporting by radiologists from anywhere. CARE was one of the first to adopt this technology through a public private partnership model with district hospital, Mahabubnagar in 2001. Over the last 18 years, we are connected to district hospitals, private centres across India, small sized hospitals and also to some international diagnostic centres in Nigeria and Iraq. This paper highlights our experiences in Teleradiology across district hospitals, private centres and international centres and also showcases the turnaround time for reporting routine and emergency cases through this mode of technology.

\section{METHODS}

All imaging modalities namely computed radiography (CR), computed tomography (CT) and magnetic resonance imaging (MRI) that were used are DICOM compliant. These machines were seamlessly integrated and configured adhering to the DICOM protocol to push images through DICOM gateway service to receive images according to set predefined standards. The PACS server had MS SQL database to host all the patient demographics along with history for each case and all images were archived based on a file system architecture. Virtual assistants help radiologists by transcribing the report online, after which another team scrutinizes for quality assurance of the reports transcribed before finally signing off the report.

\section{RESULTS}

Teleradiology network was established with 3 district hospitals (Mahabubnagar, Tandur and Nalgonda), 9 private centres (Balaji Diagnostic Centre, Matrix Hospital, Holistic Hospital, Prime Hospital, KRSNA, KIMS, Archana, Sahasra, Kiran) and 2 international centres (Mecure Diagnostic Centre, Nigeria, Medya Diagnostic Centre-Iraq). More than 1,00,000 cases have been reported using this platform. The average turnaround time for routine cases was between 4-6 hours and for all emergency cases it was between 10-30 minutes.

\section{CONCLUSIONS}

In our experience, we found Teleradiology to be an effective methodology of reporting especially if virtual assistants are used in the reporting process, thereby optimizing the radiologist time. The turnaround time for cases significantly improved especially for emergency cases.

\section{KEY WORDS}

DICOM (Digital Imaging and Communications in Medicine), PACS (Picture Archiving and Communication System), MBNR (Dist. Hospital Mahbubnagar), TNDR (Dist. Hospital Tandur), CR (Computed Radiography), CT (Computed Tomography), MRI (Magnetic Resonance Imaging), Bandwidth, Turnaround Time, Artificial Intelligence (AI)

HOW TO CITE THIS ARTICLE: Belman M, Toka M, Raghupathi NT, et al. Teleradiology - easy accessibility of radiological services for diagnosis- our experience. J. Evolution Med. Dent. Sci. 2019;8(27):2144-2148, DOI: 10.14260/jemds/2019/470

'Financial or Other Competing Interest': None.

Submission 12-03-2019, Peer Review 22-06-2019,

Acceptance 28-06-2019, Published 08-07-2019.

Corresponding Author:

Dr. Murali Belman,

\#702 A, Road No. 36,

Jubliee Hills, Hyderabad-500033, Telangana, India.

E-mail: drbelmanmurali@gmail.com

DOI: $10.14260 /$ jemds $/ 2019 / 470$

\section{(c) $(1) \ominus$}

\section{BACKGROUND}

There have been significant changes in the field of Radiology over the last two decades especially with the advances made in the Information and Communication Technology (ICT). The concept of having standardization in medical grade images by the American College of Radiology (ACR) and National Engineers Manufacturers Association (NEMA) led to a format known as Digital Imaging and Communication in Medicine (DICOM). The advent of picture archiving and communication 
system (PACS) has provided a platform wherein images from the medical equipment are sent to a server which houses a gateway application to receive DICOM images. With increased remote access to images via PACS and other patient data via the electronic health record, the changing distance between clinicians is more pervasive than just with teleradiology. Within radiology, not only has the technology changed, but the expectations of the public in response to those changes have changed as well, in part, leading to the increased subspecialization of radiologists along systems- and diseaserelated specialties.1,2 The earliest efforts in teleradiology probably date back to 1929 , when dental x-rays were transmitted with the help of telegraph to a distant location. ${ }^{3}$ Today, digitized images are transmitted around the globe via high-speed telecommunication links on a regular basis. Teleradiology has the potential to bring both positives and negatives to patient care. Radiologists have used teleradiology to simplify geographic and overnight coverage challenges as well as to strengthen subspecialty expertise. An important virtue of teleradiology is that many smaller hospitals that struggle to maintain adequate off-hour and subspecialty coverage can rapidly provide high-quality interpretations around the clock. Centralized image distribution hubs allow efficient access to qualified teleradiologists by hospitals and emergency departments needing quality reports for their imaging services. The demand for diagnostic and image interpretation services in radiology is growing rapidly in the world. This has highlighted two main issues-

1. Lack of adequate staff for interpretative coverage.

2. Lack of Specialists.

To some extent, these problems can be overcome by utilizing robust communication and image transfer systems to draw on the expertise of distantly located radiologists. This process whereby images are transferred to distant locations for the purpose of interpretation and diagnosis is termed Teleradiology. It may have started primarily in the US, but it has spread dramatically across the world. The birth of teleradiology is essentially due to the imbalance between the demand and availability of diagnostic services.

Most teleradiology practices in developed countries where digital acquisition devices are more the norm than the exception (Even in rural areas), tend to require that teleradiology images be only from digital acquisition devices. The main reason for this is that digitized images generally are of poorer quality which leads to reduced diagnostic confidence. ${ }^{4,5}$ When digitizers are used, there is evidence that for the most part digitizers are fairly equivalent in terms of acceptable contrast and low geometric distortion (even the less expensive non-radiology specific ones), thus there are circumstances where these solutions are acceptable. ${ }^{6}$ Even with digitally acquired images, however, there is considerable variability in the quality of images between sites, both as a function of the basic imaging procedures and the equipment used, so users need to be aware of these variations and account for them during the interpretation process. ${ }^{7}$ Patient privacy and data security are key issues in any radiology service, ${ }^{8}$ so teleradiology is no exception. Although security and privacy have been incorporated into teleradiology practice nearly since its inception, there continues to be considerable research in this area looking for improvement. ${ }^{9,10}$

\section{METHODS}

All imaging modalities namely computed radiography (CR), computed tomography (CT) and magnetic resonance imaging (MRI) that were used are DICOM compliant.

\section{Workflow Process}

Essentially teleradiology process involves-

1. Acquisition of images from medical equipment (CR, CT, MR)

2. Transfer of these images to a server for archival.

3. Retrieval of these images from the server for viewing.

\section{Acquisition and Transfer}

Today, virtually all radiology equipment is fully DICOM compliant. This module essentially involves configuring every modality to interact with a gateway service in the server. Once configured, images acquired from the modality get automatically pushed to the server. Lossy and lossless compression is possible; varying degrees of loss of information may be acceptable, depending upon the modality and the clinical situation. Patient demographic data and clinical history are stored in a database and images can either be stored in the database or can follow a folder system architecture in the server. Many teleradiological applications follow the folder system architecture and store only the clinical information in the database. This acquisition of cases through the DICOM Gateway works in a Spoke-hub model i.e. the central server can receive cases from any of the physical locations across different geographical areas of any given health care facility.

\section{Viewing Module}

The viewing module comprises of a DICOM viewer which displays the images and provides tools for post processing of the image such as grey shade levelling, calculation of area and volume of images, Hounsfield unit, window levelling and converting DICOM images to various other picture and video file formats.

The cases are displayed in the list according to the time they are sent in a First In-First out manner. STAT (Short Turn Around Time) cases are colour coded(red) differently so that they are easily noticeable by the user and can be reported immediately. The cases also show the received time stamp so that they can be reported accordingly.

\section{Reporting Module}

This module helps in generation of report by the doctor which in turn is sent to healthcare facility. Typically the doctor after seeing the image and forming an opinion, sends the feedback to the patient in a report format. This report is tagged along with the image so that the patient can use it as a reference whenever he is consulting specialist physician for his clinical problem.

Various report generator tools are incorporated into our solution which are helpful for the doctors to generate the report in quick and efficient way. Various formats are provided for each organ in an illustrative way, the doctors will use these formats and do further modifications in the template as needed and will generate the final report. 


\begin{tabular}{|c|c|c|}
\hline Centre Description & Total No. of Cases & $\mathbf{\%}$ \\
\hline District Hospitals & 75403 & 52 \\
\hline Private Diagnostic Centres & 40321 & 28 \\
\hline International Centres & 28549 & 20 \\
\hline \multicolumn{2}{|c|}{ Table 1. Case List }
\end{tabular}

\begin{tabular}{|c|c|}
\hline Dist. Hospitals & Total \\
\hline Mahabubnagar & 41910 \\
\hline Tandur & 17492 \\
\hline Nalgonda & 16001 \\
\hline \multicolumn{2}{|c|}{ Table 2. Govt. Centres } \\
\hline
\end{tabular}

\begin{tabular}{|c|c|c|}
\hline Centre & Description & Number \\
\hline \multirow{3}{*}{$\begin{array}{c}\text { Mahabubnagar } \\
\text { (Jan. 13-Dec. 15) }\end{array}$} & Brain & 7859 \\
\cline { 2 - 3 } & Chest & 84 \\
\cline { 2 - 3 } & Abdomen & 133 \\
$\begin{array}{c}\text { Tandur } \\
(2013-2019)\end{array}$ & Brain & 12063 \\
\cline { 2 - 3 } & Chest & 544 \\
\cline { 2 - 3 } & Abdomen & 4219 \\
\cline { 2 - 3 } & Spine & 320 \\
\hline \multirow{2}{*}{$\begin{array}{c}\text { Nalgonda } \\
(2017-2019)\end{array}$} & PNS & 162 \\
\cline { 2 - 3 } & Brain & 86 \\
\cline { 2 - 3 } & Chest & 70 \\
\hline \multicolumn{2}{|c|}{ Table 3. Study Wise Distribution } \\
\hline
\end{tabular}

\begin{tabular}{|c|c|c|c|c|}
\hline \multirow{2}{*}{ Center Name } & \multicolumn{3}{|c|}{ Modality } & \multirow{2}{*}{ Total } \\
\cline { 2 - 4 } & CR & CT & MR & \\
\hline Mecure Diagnostics, Nigeria & 17916 & 5927 & 2432 & 26275 \\
\hline Medya Diagnostics, Iraq & 412 & 823 & 1039 & 2274 \\
\hline Table 4. Cases from International Centres \\
\hline
\end{tabular}

\begin{tabular}{|c|c|c|c|c|c|c|}
\hline \multicolumn{7}{|c|}{$\begin{array}{c}\text { Average Turnaround Time } \\
\text { (Rounded off to the Nearest) }\end{array}$} \\
\hline & MBNR & TNDR & NALG & BALAJI & MATRIX & KIMS \\
\hline Routine Cases (hours) & 6.5 & 5 & 5.5 & 2 & 3.5 & 3 \\
\hline Emergency Cases (minutes) & 20 & 15 & 20 & 10 & 20 & 15 \\
\hline \multicolumn{7}{|c|}{ Table 5. Turnaround Time for Reporting } \\
\hline "This data is with reference to only CT BRAIN cases from all these centres. \\
\hline
\end{tabular}

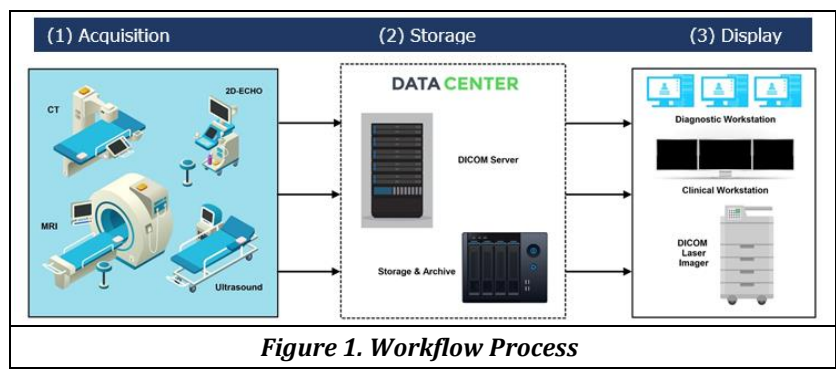

The application architecture for the teleradiology solution is shown in the diagram below-

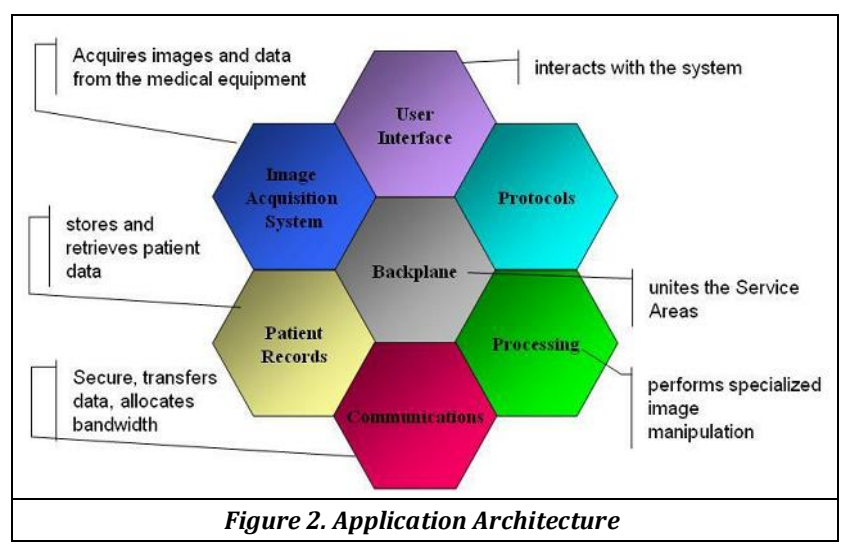

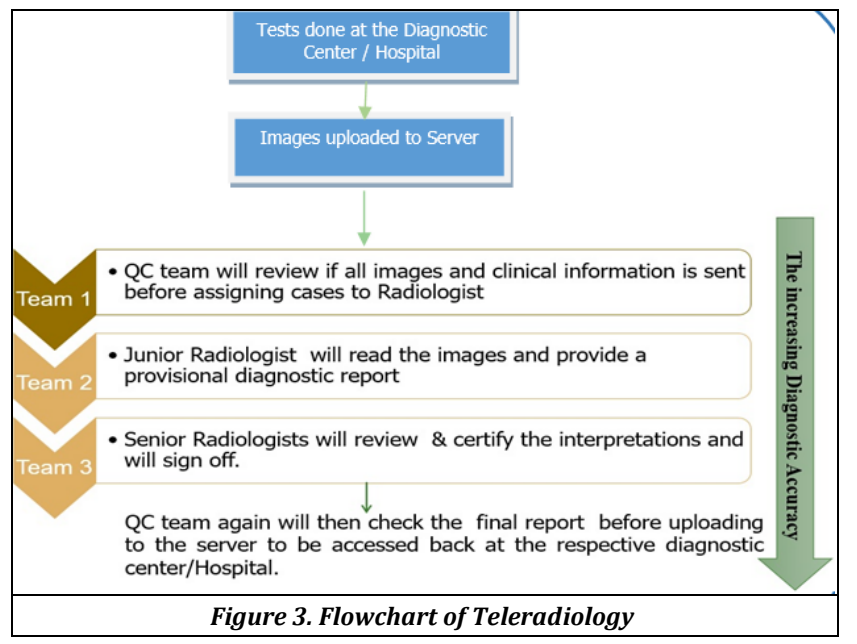
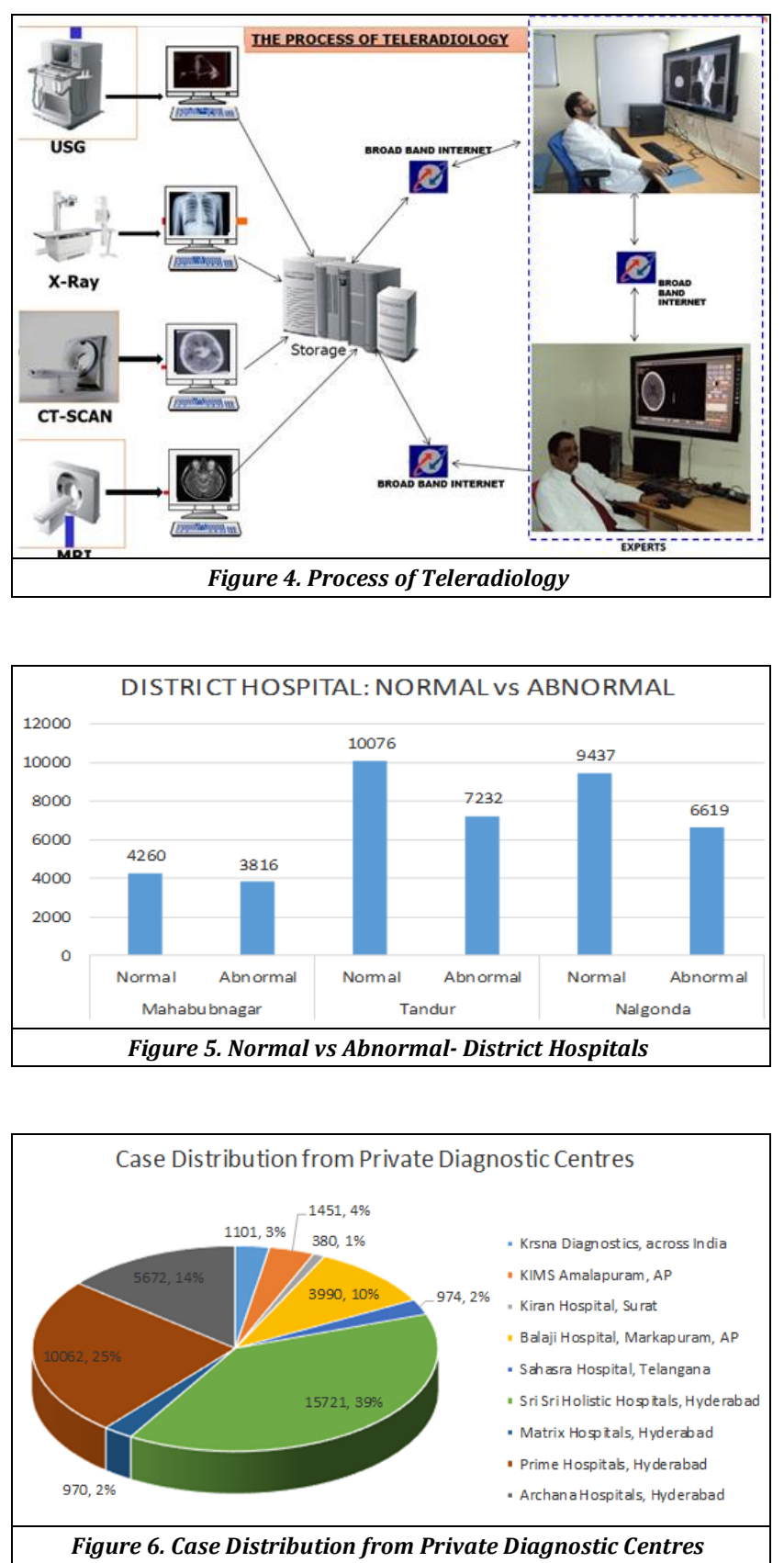


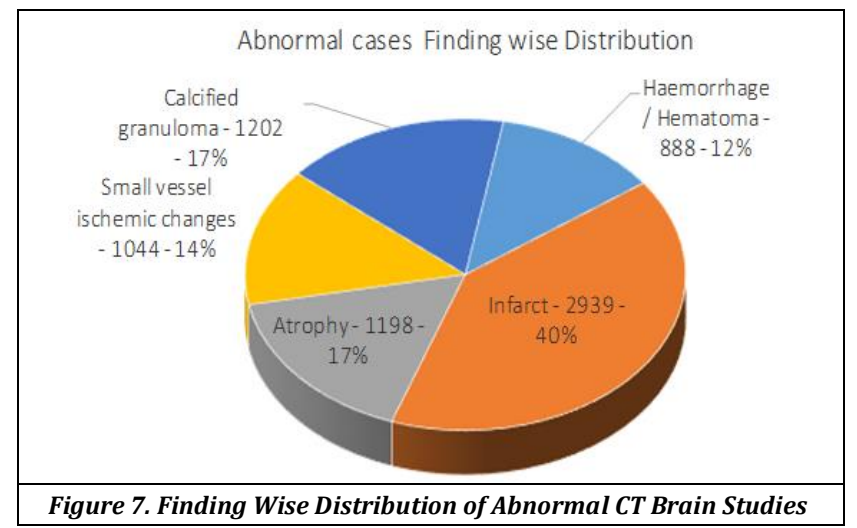

These reporting tools are designed in such a way that a report can be generated by few clicks in most cases and only simple modifications are needed in most cases. This is achieved by taking into account most of the abnormal conditions for each organ and adding specific sentences into the report for these organs based on the clicks which doctor the makes. This abnormal sentence for this organ replaces the normal sentence and is appended with all other features which are normal.

The basic design of the system satisfies the following-

- Is user-friendly and is easy operability

- To provide application on mobile and tablet platforms and make it cross-platform compatible.

- Application Conformance-HL7

- Medical Images-DICOM standard

There are some important considerations to keep in mind before adopting the process of teleradiology-

1. Final versus preliminary reads (cost and turnaround times would differ) as preliminary reading may be given by a junior radiologist.

2. Time based coverage or $24 / 7$ coverage.

3. To specify the turnaround time of reporting for emergency cases and the workflow process for such cases.

\section{RESULTS}

Teleradiology network was established with 3 district hospitals (Mahabubnagar, Tandur and Nalgonda), 9 private centres (Balaji diagnostic centre, Matrix hospital, Holistic hospital, Prime hospital, KRSNA, KIMS, Archana, Sahasra, Kiran) and 2 international centres (Mecure Diagnostic centreNigeria, Medya diagnostic centre-Iraq). A central team of radiologists were identified for reporting of the cases from any geographic location. Having a good speed of internet (2 Mbps) was the only requirement apart from the application software for viewing of images and reporting the same. One of the key indicators in establishing Teleradiology is the turnaround time achieved. With now the flexibility of viewing images from anywhere by the radiologists, this methodology has ensure shorter turnaround times and also the option of reassigning cases to other radiologists on network if cases have not been reported by a specified time. Table 1 gives us the number of cases diagnosed across three sectors namely (i) District Hospitals, (ii) Private diagnostic centres/small size hospitals across India and (iii) International centres namely Nigeria and Iraq.
Table 2 gives us the list of cases from the district hospitals of Mahabubnagar, Tandur (2013-2019) and Nalgonda (20172019).

Figure 5 gives us a list of normal vs abnormal cases from the district hospitals. The data highlights a sizable percentage, more than $50 \%$ in all three district centres of abnormal cases.

Table 3 indicates the list of CT cases done. CT Brain was identified to be the most common diagnostic test, possibly because of the proximity of the district hospitals to national highways, leading to a number of trauma related incidents.

Figure 6 indicates the list of cases from private diagnostic centres. These included CR, CT and MR cases.

Teleradiology was also established with two international centres one at Nigeria and the other at Iraq. Table 4 shows the list of cases done with these two centres for a period of 1.5 years (June 2009-December 2010) and 6 months (0 respectively.

CT Brain was observed to be the main diagnostic test across centres from where Teleradiology was done. A broad classification is indicated in Figure 7 of the CT Brain cases from selected centres (MBNR (Jan 2013-Dec 2015), Tandur (2013-2019), Nalgonda (2017-2019), Balaji (2017-2019)).

The average turnaround time for reporting with the use of this technology is indicated in Table 5 below.

\section{DISCUSSION}

As the patient clientele is more in district hospitals than in private centres because of the costs involved, Teleradiology appeared to be an effective option in addressing the issue of dearth of radiologists as the platform provides the opportunity to have cases reviewed by radiologists from any location. It also provides the accessibility to get second opinion of the same because of the architecture with which the application software is developed. Most district hospitals are located closed to national highways wherein trauma cases appear in large numbers. Diagnosing of critical cases like brain haemorrhage with short turnaround time has become possible with this technology thereby facilitating in initiation of treatment either at the district centre or in shifting the patient to city hospitals for treatment. In our experience, we found that this can be very effective methodology of reporting especially if virtual assistants are used in the process. Virtual assistants could be located anywhere as long as they have access to the application. Radiologists view images and record their findings either by audio or by indicating a macro-based template. The virtual assistant transcripts the audio or decodes the macro indicated and keys in the report. The turnaround time for cases significantly improved adopting this methodology as it delinked the radiologist time with the transcription of the report. Today with mobile based application, cloud storage and good internet speeds available across India, this technology has gained widespread implementation. However, the low pricing of radiology studies and the easy availability of the application software has led to a stiff competition amongst vendors providing these services, sometimes leading to compromise in the quality of reporting because of associated costs in radiology fee. One of the most important benefits that this technology offers is in the development of algorithms for artificial intelligence. With teleradiology being widely adopted, a platform has been created wherein images are 
available easily for development of algorithms leading to the usage of artificial intelligence (AI).

\section{CONCLUSIONS}

The dearth of radiologists and thereby difficulty in reporting of cases has been well addressed by the adaptation of Teleradiology. Teleradiology has become embedded in the health care system and integrated into a wide variety of radiology practices and will likely continue to be the standard of care for many sites where onsite radiology is either limited or lacking completely. However, there are concerns also especially in terms of the costing for providing these services and legality. Artificial intelligence (AI) will increase the efficiency of radiologists even further because of alerts that AI generates making reporting comprehensive and with less medical errors. In specific AI based algorithms can be especially very useful for diagnostic centres at remote centres where there is a difficulty in the availability of radiologist. These algorithms can indicate a risk score based on the images acquired to prompt the technicians to alert patients/patient dependants, the emergency involved and trigger the triage process for further treatment well in advance than waiting for radiologist report.

\section{REFERENCES}

[1] Silva E 3rd, Breslau J, Barr RM, et al. ACR white paper on teleradiology practice: a report from the Task Force on Teleradiology Practice. J Am Coll Radiol 2013;10(8):575-85.
[2] European Society of Radiology 2009. The future role of radiology in healthcare. Insights Imaging 2010;1(1):211.

[3] Sending dental X-rays by telegraph. Dent Radiogr Photogr 1929;2:16.

[4] Hunter TB, Krupinski EA, Weinstein RS. Factors in the selection of a teleradiology provider in the United States. J Telemed Telecare 2013;19(6):354-9.

[5] Krupinski EA, McNeill K, Ovitt TW, et al. Patterns of use and satisfaction with a university-based teleradiology system. J Digit Imaging 1999;12(2 Suppl 1):166-7.

[6] Salazar AJ, Camacho JC, Aguirre DA. Comparison between different cost devices for digital capture of $\mathrm{X}$ ray films: an image characteristics detection approach. J Digit Imaging 2012;25(1):91-100.

[7] Abramson RG. Variability in radiology practice in the United States: a former teleradiologist's perspective. Radiology 2012;263(2):318-22.

[8] Relyea-Chew A. Major regulatory changes and the impact on diagnostic imaging in the United Sates 2005 to 2012. Acad Radiol 2013;20(9):1063-8.

[9] Satoh H, Niki N, Eguchi K, et al. Teleradiology network system on cloud using the web medical image conference system with a new information security solution. Proc SPIE Med Imaging 2013;8674:86740X1.

[10] Nyeem H, Boles W, Boyd C. A review of medical image watermarking requirements for teleradiology. J Digit Imaging. J Digit Imaging 2013;26(2):326-43. 